\title{
Laparoscopic Gastroscopic Transgastric Cystogastrostomy and Cholecystectomy for Pseudopancreatic Cyst after Gallstone Pancreatitis in Children
}

\author{
Bethany J. Slater ${ }^{1} \quad$ Ashwin Pimpalwar ${ }^{1}$ \\ ${ }^{1}$ Division of Pediatric Surgery, Michael E Debakey Department of \\ General Surgery, Baylor College of Medicine and Texas Children \\ Hospital, Houston, Texas, United States \\ Eur J Pediatr Surg Rep 2014;2:10-12.
}

\begin{abstract}
Address for correspondence Ashwin Pimpalwar, MD, FRCS, Division of Pediatric Surgery, Texas Children Hospital, 3765 Drummond Street, Houston, TX 77025, United States (e-mail: ashwinpgi@hotmail.com; appimpal@texaschildrenshospital.org).
\end{abstract}

$$
\begin{aligned}
& \text { Abstract } \\
& \text { Keywords } \\
& \text { - laparoscopic } \\
& \text { cystogastrostomy } \\
& \text { - endoscopic } \\
& \text { cystogastrostomy } \\
& \text { - cystogastrostomy } \\
& \text { - pancreatic } \\
& \text { pseudocyst } \\
& \text { - cyst of pancreas }
\end{aligned}
$$

A 15-year-old girl presented with gallstone pancreatitis. Subsequently, a pseudopancreatic cyst developed that was diagnosed on computed tomographic scan. She underwent a laparoscopic and gastroscopic transgastric cystogastrostomy. In the following report, we describe our novel approach and technique for the above condition.

\section{Introduction}

Several techniques have been described for the management of pseudopancreatic cysts. These include open, laparoscopic, or endoscopic approaches. However, there are disadvantages and limitations with each of these techniques. The open method requires a large incision with its associated morbidity, and the laparoscopic techniques described so far are technically challenging in the pediatric population. The endoscopic approach has poor results as the cystenteric opening is small and tends to occlude leading to recurrence and infection. We describe our combined technique of laparoscopic and gastroscopic transgastric cystogastrostomy for pseudopancreatic cysts in children.

\section{Case Report}

A 15-year-old girl presented to the emergency room with abdominal pain. She was diagnosed with gallstone pancreatitis. She had high amylase and lipase levels and a computed tomographic (CT) scan showed necrotizing pancreatitis. She was managed conservatively for 7 days. She was then discharged home. She presented to the ER after 6 weeks with abdominal pain and vomiting. Her amylase and lipase were grossly elevated. Subsequently, an abdominal CT scan revealed a well-formed wall enhancing pseudopancreatic cyst (-Fig. 1). She was then taken to the operating room for surgical management.

\section{Technique}

Under anesthesia a pediatric Pentax gastroscope (Pentax Medical Company, Montvale, New Jersey, United States) was introduced into the stomach. A 5-mm step port was placed in the umbilicus. Three more 5-mm step ports were placed under direct vision: 2 ports on either side of the umbilicus and 1 port just under the costal arch to retract the gall bladder. Using these ports, a cholecystectomy was performed in the standard fashion. The stomach was insufflated with air from the gastroscope and two sutures of 3-0 vicryl were placed to pexy the stomach to the abdominal wall using a Granee needle (R-Med, received

August 10, 2013

accepted after revision

August 26, 2013

published online

December 12, 2013

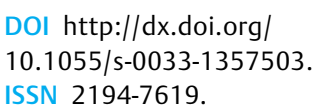

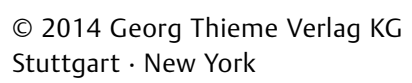

License terms

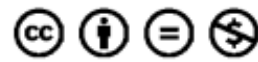




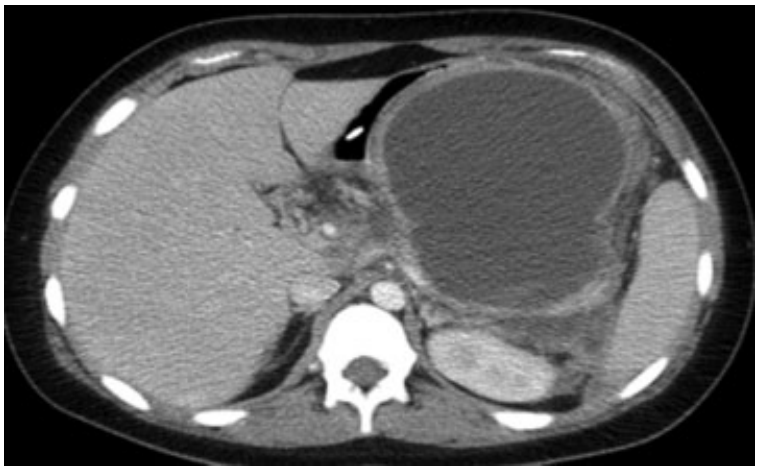

Fig. 1 Axial cut of computed tomographic scan demonstrating pseudocyst posterolateral to stomach.

Oregon, Ohio, United States) ( $=$ Fig. 2A). A 12-mm port was then directly introduced into the stomach under vision of the gastroscope as well as the laparoscope ( - Fig. 2B). It is very important to have the visualization through the gastroscope while placing the initial transgastric port as the cyst protruding from the posterior gastric wall compromises the gastric lumen severely. If performed without the visualization of the gastroscope there is a risk injury to the posterior gastric wall during the transgastric port placement. One of the other ports used for gallbladder removal was then also introduced into the stomach under vision. The 5-mm 30-degree telescope was then introduced into the stomach and the stomach was continuously insufflated with low flow. The pseudocyst was easily identified on the posterior wall of the stomach. A laparoscopic needle was introduced through the laparoscopy port into the cyst to confirm the position of the cyst (-Fig. 3A). A harmonic scalpel was then introduced into the stomach through the same port and an opening was made into the posterior wall of the stomach in to the cyst (-Fig. 3B). Once this was achieved a 30-mm Endo GIA (Covidien, Mansfield, Massachusetts, United States) stapler was introduced through the 12-mm umbilical port and a stapled cystogastrostomy was achieved (-Fig. 3C, D). The cyst contents were suctioned and the cyst was irrigated with saline. Gastric port sites were closed with 3-0 vicryl interrupted sutures.

\section{Results}

The patient tolerated the procedure well with no intraoperative complications and was started on a regular diet on the fourth postoperative day. She was discharged home on the sixth postoperative day.

She was followed up in clinic at 4 weeks and 3 months postoperatively. She had well-healed scars and was tolerating a regular diet with no other problems.

\section{Discussion}

Pancreatic pseudocysts, because of pancreatitis, trauma, or ductal obstruction, are uncommon in children. ${ }^{1}$ However, they may require treatment if symptomatic or persist after 4 to 6 weeks. Internal drainage of pancreatic pseudocysts has traditionally been accomplished by laparotomy, although laparoscopic and endoscopic modalities have recently been described. The open technique has been shown to have mortality rates of 1 to $6 \%$, complication rates of 10 to $40 \%$, and recurrence rates of 5 to $20 \%{ }^{2}$

The laparoscopic technique consists of either creation of an anterior gastrotomy to create a psuedocyst gastrostomy or identification of the cyst stomach interface through the lesser sac. ${ }^{3}$ Both modalities have been shown to be safe and effective in the adult literature but both can be technically challenging and cumbersome in the smaller working space in children.

Endoscopic therapy alone involves identifying the region of the cyst bulge with the endoscope, needle localization of the tract, and stent placement for internal drainage. However, the cystenteric opening is limited in size which can lead to occlusion, infection, or recurrence. ${ }^{4,5}$ Beckingham et al performed endoscopic drainage in 34 adults with a success rate of $71 \%$. The failures were found to be associated with pseudocysts complicated by acute necrotizing pancreatitis and cyst wall thickness greater than $1 \mathrm{~cm} .{ }^{6}$ Repeated procedures due to catheter-related complications requiring additional anesthetics are not ideal in the pediatric population. Melman et al compared the three techniques for drainage of pancreatic pseudoscyts in adults and found lower primary success rates for endoscopic drainage as compared with laparoscopic and
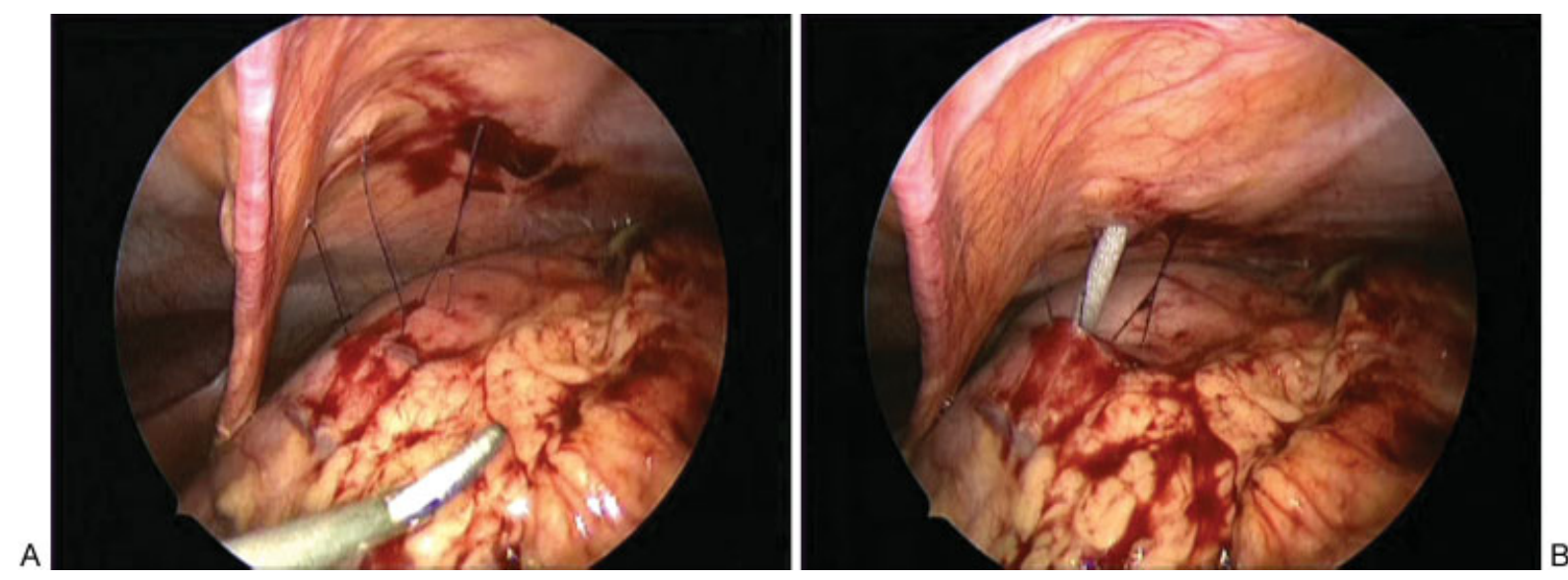

Fig. 2 (A) Pexy sutures placed on the anterior gastric wall. (B) Step port directly introduced into the stomach. 

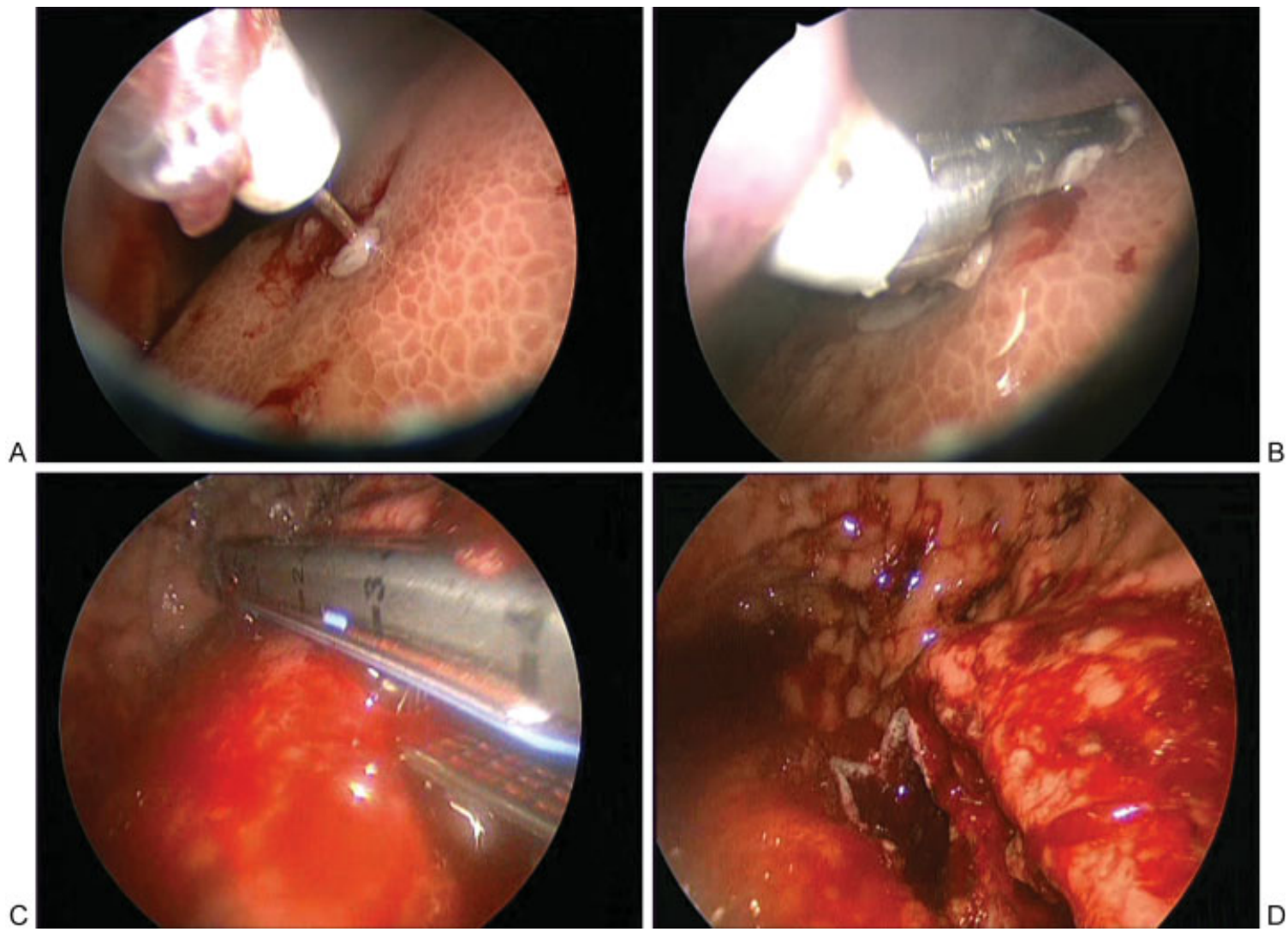

Fig. 3 (A) Laparoscopic needle inserted in the posterior gastric wall to confirm the presence of pseudocyst. (B) Harmonic scalpel used to incise the posterior wall of stomach and the pseudocyst after needle aspiration of the pseudocyst. (C) Endo GIA stapler being applied to the posterior gastric wall and the pseudocyst. (D) Endo GIA stapler applied to the posterior gastric wall and the pseudocyst.

open cystgastrostomies as well as the need for repeat procedures. ${ }^{5}$

The approach of combined laparoscopic and endoscopic/ gastroscopic pseudocyst gastrostomy using intraperitoneal and as well as intragastric visualization has been described in the adult literature but has not been previously reported in children. ${ }^{4,7}$ The use of the laparoscopy during cystgastrostomy allows for pseudocyst debridement, fashioning a large communication between the cyst and stomach, as well as minimizes the risk of complications such as gastric perforation. In addition, laparoscopy adds the ability to add other procedures if needed such as cholecystectomy as was done in this case. Gastroscopy helps in visualization of the primary transgastric port placement preventing injury to the posterior gastric wall and gastric insufflation during the procedure. Finally, the decreased need for additional port sites leads to an improved cosmetic result which is of particular interest in the pediatric population.

\section{Conclusion}

Laparoscopic Gastroscopic Transgastric Cystogastrostomy is a safe technique for the treatment of pancreatic pseudocysts in children. We recommend the use of this technique in suitable cases.

\section{Conflict of Interest}

None.

\section{References}

1 Teh SH, Pham TH, Lee A, Stavlo PL, Hanna AM, Moir C. Pancreatic pseudocyst in children: the impact of management strategies on outcome. J Pediatr Surg 2006;41(11):1889-1893

2 Bergman S, Melvin WS. Operative and nonoperative management of pancreatic pseudocysts. Surg Clin North Am 2007;87(6): 1447-1460, ixix.

3 Park AE, Heniford BT. Therapeutic laparoscopy of the pancreas. Ann Surg 2002;236(2):149-158

4 Ammori BJ, Bhattacharya D, Senapati PS. Laparoscopic endogastric pseudocyst gastrostomy: a report of three cases. Surg Laparosc Endosc Percutan Tech 2002;12(6):437-440

5 Melman L, Azar R, Beddow K, et al. Primary and overall success rates for clinical outcomes after laparoscopic, endoscopic, and open pancreatic cystgastrostomy for pancreatic pseudocysts. Surg Endosc 2009;23(2):267-271

6 Beckingham IJ, Krige JE, Bornman PC, Terblanche J. Long term outcome of endoscopic drainage of pancreatic pseudocysts. Am J Gastroenterol 1999;94(1):71-74

7 Atabek U, Mayer D, Amin A, Camishion RC. Pancreatic cystogastrostomy by combined upper endoscopy and percutaneous transgastric instrumentation. J Laparoendosc Surg 1993;3(5):501-504 